\title{
Mitteilungen der DGKFO
}

J Orofac Orthop 2021 $\cdot 82: 282-286$

https://doi.org/10.1007/s00056-021-00308-z

() Springer Medizin Verlag GmbH, ein Teil von

Springer Nature 2021

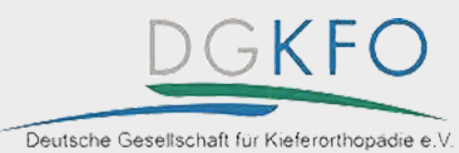

\section{What's new}

\section{Inhalt}

282 What's new

283 Buchbesprechung: Checklisten der Zahnmedizin: Kieferorthopädie

284 Nachruf

285 Personalia

285 Tagungen
Wirksamkeit von Verfahren zur Reduzierung von Bioaerosolen in der Zahnmedizin: eine systematische Übersichtsarbeit

\section{Originalpublikation}

Samaranayake LP, Fakhruddin KS, Buranawat B, Panduwawala C (2021) The efficacy of bio-aerosol reducing procedures used in dentistry: a systematic review. Acta Odontol Scand. 79(1):69-80

\section{Hintergrund}

Bioaerosole werden in der klinischen Zahnmedizin routinemäßig erzeugt und in die Luft getragen, da die operativen Instrumente in einer oralen Umgebung eingesetzt werden, die von Speichelorganismen durchdrungen ist. Die SARS-CoV-2-Übertragung, die für die derzeitige Pandemie verantwortlich ist, erfolgt über Aerosole und Tröpfchen in der Luft, weshalb man sich intensiv mit solchen Aerosolen erzeugenden Verfahren und deren Reduzierung beschäftigt hat.

\section{Ziel}

Ziel dieser systematischen Übersichtsarbeit war es, die verfügbaren Daten zu drei Hauptmaßnahmen zu bewerten: Anlegen von Kofferdam, präprozedurale Mundspülung und hochvolumige Absaugung (HVE) zur Reduzierung von Bioaerosolen.

\section{Material und Methode}

Eine elektronische Datensuche von englischsprachigen Manuskripten unter Verwendung der Datenbanken Ovid MEDLINE, Web of Science, EBSCO host und Cochrane Library wurde von 2 Untersuchern durchgeführt. Es wurde auf veröffentlichte klinische Berichte zwischen dem 01. Januar 1985 und dem 30. April
2020 zugegriffen. In den Datenbanken wurden klinische Studien zu KofferdamBioaerosol, HVE-Bioaerosol und präprozeduraler Mundspülung identifiziert.

\section{Ergebnisse}

Insgesamt wurden 156 Einträge in der englischsprachigen Literatur identifiziert, 17 klinische Studien mit 724 Patienten wurden in die endgültige Analyse aufgenommen. Die infrage kommenden Artikel zeigten die Unzulänglichkeit von 3 prinzipiellen Ansätzen, die in der zeitgenössischen zahnärztlichen Praxis zur Minimierung solcher Bioaerosole verwendet werden: die Anwendung von Kofferdam, präprozedurale Mundspülungen und HVE. Letztere ist eine äußerst effektive Methode zur Reduktion von Bioaerosolen in der Zahnmedizin, obwohl keine einzelne Methode eine vollständige Wirksamkeit hat.

\section{Schlussfolgerung}

Die vorliegende systematische Übersichtsarbeit zeigt, dass der Einsatz von Kombinationsstrategien aus Kofferdam, einer präprozeduralen antimikrobiellen Mundspülung und insbesondere der HVE Bioaerosole während operativer Eingriffe eindämmen kann. 


\section{Dentalaerosole: Mikrobielle Zusammensetzung und räumliche Verteilung}

\section{Originalpublikation}

Zemouri C, Volgenant CMC, Buijs MJ, Crielaard W, Rosema NAM, Brandt BW, Laheij AMGA, De Soet JJ (2020) Dental aerosols: microbial composition and spatial distribution. J Oral Microbiol 12(1):1762040

\section{Hintergrund}

Hochtourige zahnärztliche Instrumente erzeugen Aerosole, die zur Übertragung von pathogenen Mikroorganismen beitragen können.

\section{Ziel}

Ziel dieser Studie ist es, die mikrobielle Belastung, die Zusammensetzung und die räumliche Verteilung von Aerosolen in Zahnkliniken zu beschreiben.

\section{Material und Methode}

In 4 Zahnkliniken wurden an verschiedenen Orten vor, während und nach der Behandlung aktive und passive Probenentnahmen vorgenommen. Die erhaltenen koloniebildenden Einheiten (KBE) wurden zur Taxonidentifizierung sequenziert.

\section{Ergebnisse}

Die Proben enthielten bis zu $655 \mathrm{KBE} /$ Platte $/ 30$ min und $418 \mathrm{KBE} / \mathrm{m}^{3} / 30 \mathrm{~min}$ während der zahnärztlichen Behandlung. Der Kontaminationsgrad nach der Behandlung und in 1,5 m Entfernung vom Kopf des Patienten war ähnlich wie zu Beginn des Tages. Die höchste Kontamination wurde im Brustbereich des Patienten festgestellt. Die Aerosole setzten sich aus 52 verschiedenen Taxa menschlichen Ursprungs und 36 aus Wasser zusammen.

\section{Schlussfolgerung}

Die bakterielle Kontamination durch Tröpfchen ist während der zahnärztli- chen Behandlung von Bedeutung. In einem Abstand von 1,5 m von der Mundhöhle wurde kein Anstieg der bakteriellen Kontamination festgestellt. Es gab jedoch innerhalb von $80 \mathrm{~cm}$ um den Kopf des Patienten eine hohe Kontamination mit Taxa sowohl menschlichen als auch wässrigen Ursprungs. Die Ergebnisse unterstreichen die Bedeutung von Infektionskontrollmaßnahmen in zahnärztlichen Wasserleitungen und auf Oberflächen in unmittelbarer Nähe zum Kopf des Patienten.

\section{Korrespondenzadresse}

Prof. Dr. Helge Fischer-Brandies

Klinik für Kieferorthopädie,

Universitätsklinikum Schleswig-Holstein

Arnold-Heller-Straße, 24105 Kiel, Deutschland

Helge.Fischer-Brandies@uksh.de

Interessenkonflikt: $\mathrm{H}$. Fischer-Brandies gibt an, dass kein Interessenkonflikt besteht.

\section{Buchbesprechung}

\section{Checklisten der Zahnmedizin: Kieferorthopädie}

\author{
Harzer W (2021) Checklisten Zahn- \\ medizin: Kieferorthopädie. Thieme, \\ Stuttgart, 2. Auflage, ISBN-10 \\ 3132442186
}

Geschrieben von Prof. Winfried Harzer fasst die zweite Auflage der Checklisten der Zahnmedizin: Kieferorthopädie die wesentlichen kieferorthopädischen Aspekte auf 393 Seiten ausgezeichnet zusammen. Entsprechend dem bewährten didaktischen Konzept der Checklisten-Lehrbuchreihe geht es vor allem darum, die wichtigsten Aspekte der kieferorthopädischen Diagnostik und Therapie prägnant auf den Punkt zu bringen und mithilfe von über 700 Abbildungen und Grafiken besonders anschaulich einprägsam zu machen. Ergänzt wird dieses übersichtliche Format durch zahlreiche Fallbeispiele, die einen guten klinischen Bezug herstellen und noch mehr Greifbarkeit schaffen.

Das Lehrbuch enthält 13 Überkategorien, lässt sich jedoch grob in 4 Hauptteile gliedern, die jeweils in Kapitel aufgeteilt sind.

Der erste Teil versteht sich als eine Einleitung und beschäftigt sich mit der Entwicklung und den Aufgaben der Kieferorthopädie, den biologischen Grundlagen sowie der Ätiologie von Dysgnathien. In dieser Ausgabe neu hinzugekommen ist ein Kapitel über den immer mehr an Bedeutung zunehmenden Bereich der Digitalisierung in der Kieferorthopädie.

Der zweite Abschnitt widmet sich der kieferorthopädischen Diagnostik. Auf eine didaktisch wertvolle Art wird der Leser sehr systematisch vom kieferorthopädischen Befund bis hin zum Therapieplan

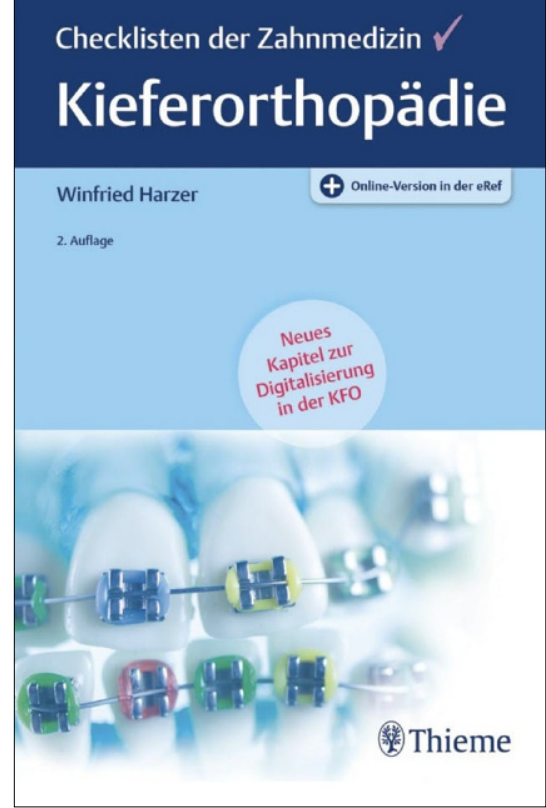


geleitet. Behandelt werden alle Aspekte der Diagnostik, beginnend mit der allgemeinen und speziellen Anamnese über die klinische, funktionelle und bildgebende Untersuchung bis hin zur Modellanalyse.

Der nächste große Abschnitt handelt von der kieferorthopädischen Therapie. Hier wird zunächst auf die biologischen Grundlagen der Zahnbewegung eingegangen, einschließlich Aspekte der Biomechanik. Nach einem Kapitel über die kieferorthopädischen Materialien werden dann sehr praxisbezogen alle wesentlichen Apparaturen und Behandlungsmethoden erläutert. Dem Thema skelettaler Verankerungsmöglichkeiten wird besondere Bedeutung beigemessen.

Konkret werden systematische Behandlungsempfehlungen für jede Malokklusion bzw. Fehlstellung angeboten, die der Leser sehr strukturiert und einfach nachvollziehen kann.
Ein weiterer Teil der Checklisten der Zahnmedizin: Kieferorthopädie beschäftigt sich schließlich mit speziellen kieferorthopädischen Themen. Dazu zählen interdisziplinäre Behandlungen, z. B. für Patienten mit kraniofazialen Fehlbildungen, Patienten in der Erwachsenenorthodontie oder mit obstruktivem Schlafapnoesyndrom. Dank der zahlreichen Merke-, Cave- und Ablaufboxen sind die Informationen auf einen Blick schnell ersichtlich und wiederauffindbar. Besonders praktisch ist die Möglichkeit, alle Inhalte des Buches ebenfalls kostenlos online nutzen zu können. Zu diesem Zweck erhält der Leser im Buch einen Bar- sowie Zugangscode, mit welchem der Zugang zur Online-Plattform eRef freigeschaltet wird.

Insgesamt ist das Buch im Vergleich $\mathrm{zu}$ anderen Lehrbüchern kompakter und zielgerichteter. Als Teil der ChecklistenLehrbuchreihe hat es selbst nicht den An- spruch, die Breite einer Lehrbuchdarstellung zu erfüllen, sondern vielmehr, dem Leser das Grundverständnis und die Fähigkeit zum Handeln zu vermitteln.

Seine schöne und einfache Struktur sowie die evidenzbasierten Zusammenfassungen der Kieferorthopädie machen dieses Buch zu einem absolut wertvollen Überblick über die wichtigen kieferorthopädischen Themen. Es ist daher sowohl für Weiterbildungsassistenten der Kieferorthopädie als auch für kieferorthopädisch tätige Zahnärzte und Studierende der Zahnmedizin als Examensvorbereitung geeignet.

\section{Korrespondenzadresse}

\section{Dr. Sema Safi}

Poliklinik für Kieferorthopädie

Welschnonnenstr. 17, 53111 Bonn,

Deutschland

\section{Nachruf}

\section{o.Univ.-Prof. Dr. Hans Peter Bantleon}

Am 23. April ist Hans Peter Bantleon nach mit bewundernswerter Akzeptanz getragener Krankheit im viel zu jungen Alter von 70 Jahren verstorben.

Nach dem Studium der Humanmedizin und Ausbildung zum Facharzt für Zahn-, Mund- und Kieferheilkunde an der Universitätsklinik in Graz begann seine klinische Laufbahn mit einer zweijährigen Tätigkeit an der Abteilung für Mund-, Kiefer- und Gesichtschirurgie bevor er 1982 zur Abteilung für Kieferorthopädie wechseln konnte. Dies war der Startpunkt einer lebenslangen von Begeisterung getragenen Auseinandersetzung mit unserem Fachgebiet. Es folgten die Ernennung zum Oberarzt 1987 sowie die Habilitation im Jahr 1989, die, wie konnte es anders sein, seine geliebte Biomechanik zum Thema hatte. Die zu diesem Zweck entwickelte Messapparatur ermöglichte eine bis dahin nicht dagewesene Präzision bei der Vermessung und Kalibrierung unterschiedlichster Biomechaniken, die an- schließend in der klinischen Anwendung auf ihre Wirksamkeit am Patienten überprüft wurden.

1992 führte ihn sein Weg nach Wien, als ordentlicher Universitätsprofessor für Kieferorthopädie. Mit der für ihn so typischen Energie fing er an, eine de facto kaum existierende Abteilung baulich, personell, wissenschaftlich und didaktisch aufzubauen. Seine „steirische Sturheit“, auf die er sehr stolz war, half ihm sicher in dieser oft schwierigen Zeit. Die Qualität seiner Arbeit wurde eindrucksvoll bestätigt, als die Abteilung für Kieferorthopädie in Wien im Jahr 2014 als eine der ersten Kliniken NEBEOP-zertifiziert wurde.

Die Ausbildung junger Kolleginnen und Kollegen lag ihm sehr am Herzen. Er war fordernd, verlangte Leistung und eine kritische Auseinandersetzung mit dem eigenen Wirken. Unvergessen werden vielen seiner Schülerinnen und Schüler die Dienstagsbesprechungen bleiben. Er wirkte vielleicht manchmal ungeduldig, ermöglichte und förderte gleichzeitig allerdings eine Weitung des Horizonts auch außerhalb klassischer Lehrmeinungen. Ein großer Teil der kieferorthopädischen Versorgung der österreichischen Bevölkerung wird von seinen ehemaligen Schülerinnen und Schülern qualitativ hochwertig erbracht.

Doch das Teilen von Wissen betraf nicht nur die Universität. Die jährliche Tagung in Kitzbühel, für die er als Präsident der Österreichischen Gesellschaft für Kieferorthopädie jahrelang mitverantwortlich war, ist ein Höhepunkt im Fortbildungskalender im deutschen Sprachraum. Leider war es Hans Peter Bantleon nicht mehr vergönnt, das 50-jährige Jubiläum, das Corona-bedingt verschoben hat werden müssen, gebührend feiern $\mathrm{zu}$ können. Das von ihm ins Leben gerufene International Vienna Orthodontic Symposium (IVOS), das jedes Jahr in Wien in der Adventszeit stattfand, wurde zum Inbegriff einer zentraleuropäischen kie- 
ferorthopädischen Begegnungsstätte. Ein besonderer Platz in seinem Berufsleben nahmen auch die Präsidentschaft der European Orthodontic Society (EOS) und die Ausrichtung des europäischen Kongresses im Jahr 2006 ein.

Legendär war Hans Peter Bantleons Großzügigkeit, die viele haben erfahren dürfen. Sehr gerne folgten demnach viele der besten Vortragenden seinen Einladungen. Sie genossen nicht nur eine besondere Tagungsatmosphäre, sondern auch eine persönliche Betreuung in seinen Lieblingsrestaurants.

Auch nach seiner Emeritierung im Jahr 2018 war Hans Peter Bantleon immer offen für Neues. Seine Begeisterung und sein Interesse trieben ihn bis knapp vor seinem Ableben an. Ebenso seine Aufrichtigkeit, mit der er immer wieder aufstand und auf in seinen Augen inkorrekte wis- senschaftliche Vorgänge und Interpretationen hinwies. Er war eine streitbare Person, in bestem Sinne, jemand der einen geradezu dazu zwang, sich mit ihm und dem gerade aktuellen Diskussionsthema auseinanderzusetzen und niemanden kalt ließ. Und manchmal merkten seine Diskussionspartner dann erst mit Verspätung, wie befruchtend diese Auseinandersetzungen persönlich und fachlich waren.

Bei allen didaktischen, wissenschaftlichen und organisatorischen Aufgaben kam auch die Patientenbehandlung nicht zu kurz. Hans Peter Bantleon lebte förmlich das klinische Fach Kieferorthopädie in seiner ganzen Breite. Ausgezeichnet wurde die von ihm erbrachte Behandlungsqualität u.a. durch seine Mitgliedschaft beim European Board of Orthodontists und der Angle Society of Europe.

drea Holtgrave zum 82. Geburtstag am 19.05.2021. Außerdem beglückwünschen wir Dr. Erich Schmidtmann zum 82 . Geburtstag am 08.05.2021, und schließlich
Weitere Auszeichnungen, die Hans Peter Bantleon für sein Lebenswerk erhielt, waren u. a. der Sheldon Friel Memorial Lecture der European Orthodontic Society im Jahr 2017 und der Paul-Herren-Award der Klinik für Kieferorthopädie Bern im Jahr 2019 als Anerkennung für herausragende Leistungen in der Lehre, im klinischen Bereich und in der Forschung der Kieferorthopädie. Eine besondere Erwähnung verdient die Verleihung des Österreichischen Ehrenkreuzes für Wissenschaft und Kunst I. Klasse im Jahr 2016.

Persönlich habe ich keine großzügigere Person kennengelernt, einen Menschen, der mich in den 33 Jahren die ich ihn kennen durfte, gefordert und gefördert hat. Meine Gedanken sind bei seiner Familie.

Univ.-Doz. Dr. Frank Weiland Deutschlandsberg, Österreich

wünschen wir Prof. Dr. Thomas Rakosi alles Gute zum 97. Geburtstag am 04.07.2021.

\section{Tagungen}

Bitte beachten Sie, dass weiterhin alle unten aufgeführten Angaben in Zeiten der COVID-19-Krise ungewiss sind. Die Entscheidung über das Stattfinden bzw. die Absage der jeweiligen Veranstaltung oder Tagung wird sich tagesaktuell den örtlichen Umständen entsprechend ändern können. Wir bitten unsere Leser daher um eigenständiges Informieren auf den jeweiligen Internetseiten kurz vor geplantem Beginn.

Vielen Dank für Ihr Verständnis!

\section{1}

19.09.-21.09.2021

Osaka, Japan

22.09.-25.09.2021

Wiesbaden, Germany

23.09.-25.09.2021

Regensburg, Germany 9th World Society of Lingual Orthodontics

www.wslo.org/congress

Jahrestagung der Deutschen Gesellschaft für Kieferorthopädie e.V. www.dgkfo.de (DGKFO)

Jahrestagung der Deutschen Gesellschaft für Kinderzahnheilkunde (DGKiZ) www.dgkiz.de 
14.10.-17.10.2021

Seoul, South Korea

05.11.-06.11.2021

Frankfurt am Main, Germany

11.11.-13.11.2021

Florence, Italy

18.11.-20.11.2021

Bad Homburg, Germany

04.12.-05.12.2021

Kaohsiung Lin, Taiwan

\section{2}

05.03.-12.03.2022

Kitzbühel, Austria

31.05.-05.06.2022

Limassol, Cyprus
The 54th Annual Scientific Congress of the Korean Association of Orthodontists \& The 12th World Implant Orthodontic Conference

Deutscher Zahnärztetag 2021

52nd International Congress of the Italian Society of Orthodontics

54. Jahrestagung der Deutschen Gesellschaft für Funktionsdiagnostik und -therapie in der Zahn-, Mund- und Kieferheilkunde (DGFDT)

34th Annual Meeting of the Taiwan Association of Orthodontists

50. Kieferorthopädische Fortbildungstagung der Österreichischen Gesellschaft für Kieferorthopädie

97th Congress of the European Orthodontic Society www.kao.or.kr/eng/

www.dtzt.de

www.sido.it

www.dgfdt.de

www.tao.org.tw/en

www.oegkfo.at

www.eoseurope.org 